\title{
Peer support for patients with type 2 diabetes in rural communities of China: protocol for a cluster randomized controlled trial
}

\author{
Bo Xie ${ }^{1 \dagger}$, Xiu-li Ye ${ }^{1 \dagger}$, Zi-lin Sun ${ }^{1 *}$, Min Jia ${ }^{1}$, Hui Jin ${ }^{2}$, Chang-ping Ju${ }^{1}$, Li Yao ${ }^{1}$, Carvalho Husni Da Costa De Vladmir ${ }^{1}$ \\ and Yanxiaoxiao Yang ${ }^{1}$
}

\begin{abstract}
Background: The prevalence of diabetes has been growing rapidly in developing countries. This causes devastating economic burdens and increases demands on the health care system. Therefore, there is an urgent need to find a cost-effective and multi-faceted approach for diabetes care. Peer support models provide a potentially low-cost, flexible means which complements the current existing health care services. In this way, trained peer leaders can become qualified extensions to a formal healthcare system, capable of assisting education delivery and bolstering the efforts of professional staff. As such, creating a cultural specific peer support program and determining whether it is acceptable and cost-effective in rural communities of China is crucial. This study aims to implement and evaluate biophysical and psychosocial outcomes of peer support program for people with type 2 diabetes in rural communities, and to explore the program's feasibility and sustainability in China.

Methods/Design: This study is a cluster randomised controlled trial. All consenting patients will be randomised by community staff members to receive either peer support or the control care. The data collection and analysis including social demographics, health status, psychosocial status, economic status and biomedical measures will be collected at baseline, 6 months, and 12 months. The primary indicator measured is the change in HbA1c, whereas secondary indicators include biophysical, psychosocial functioning and other lifestyle factors. Finally, economic evaluations will determine whether the program is cost effective.

Discussion: This protocol is a cluster randomized, controlled trial of group-based peer support for people with type 2 diabetes in the community settings of rural China. Results from this trial may provide evidence to the effectiveness of peer support; furthermore, they will provide valuable information concerning the acceptability and feasibility of a new approach to improve diabetes self-management among resource-constrained settings.
\end{abstract}

Trial registration: ClinicalTrials.gov Identifier: NCT02119572, April 18, 2014.

Keywords: Peer support, Diabetes, Self-management, Rural health

\section{Background}

Diabetes is a growing health problem worldwide. It is estimated that 382 million people live with diabetes around the world, and by 2035, that number will surge to 592 million [1]. Diabetes mellitus imposes a huge economic burden on national health care systems globally.

\footnotetext{
*Correspondence: sunzilin1963@126.com

${ }^{\dagger}$ Equal contributors

'Department of Endocrinology, Zhongda Hospital, Institute of Diabetes, Medical School, Southeast University, Nanjing 210009, China

Full list of author information is available at the end of the article
}

The global health expenditures for diabetes in 2030 is forecasted to increase 30\% to 34\% from 2010. Developing countries will experience a twenty-year growth rate of $67 \%$ while the developed countries will have a rate of $27 \%$ [2]. A systematic review showed that diabetes affects $6.8 \%$, or one out of every 15 people living in rural areas worldwide. From 1990-2010, the prevalence in low-middle income countries grew fourfold, while in high-income countries, the prevalence was twofold higher [3]. Furthermore, rural populations often live in worse socioeconomic conditions and have poor literacy skills 
compared with their urban counterparts [4]. As such, there is an urgent need to find innovative and effective solutions to help citizens in rural communities especially in low-middle income countries to successfully manage diabetes [5].

China has the largest number of individuals with diabetes mellitus; 113.9 million (11.6\%) adults at age 18 or older have diabetes and 493.4 million adults (50.1\%) are pre-diabetic. The prevalence of diabetes and pre-diabetes in rural areas is an estimated $10.3 \%$ and $50.9 \%$ respectively [6]. Considering the problem of a growing diabetic population, health professional shortages, and an under-financed health care system in China, a effective strategy is crucial. Providing education programs to a fast growing number of people with diabetes, particularly the poor and less-educated rural community, presents a huge challenge to Chinese health care system. To improve diabetes care and positively influence health outcomes, new approaches need to be developed that are both effective and feasible in Chinese rural communities [7].

Nowadays, peer support models have been widely recognized as a promising solution. The concept of peer support emphasizes a patient-centered approach as "the provision of emotional, appraisal and informational assistance by a created social network member who possesses experiential knowledge of a specific behavior or stressor and similar characteristics as the target population, to address a healthrelated issue of a potentially or actually stressed focal person" [8]. Although the WHO reviewed the use of peer support programs for people with diabetes and found numerous patient benefits, such as glycemic control and increase in quality of life, further evaluations are needed before peer support interventions can be integrated into the existing policy for diabetes management [9]. A systematic review by Jeremy Dale and colleagues found that peer support programs exhibit a latent potential towards improving health outcomes in adults living with diabetes. However the evidence-based review also identified the evaluation's inconsistency and limitations, emphasizing the need for additional data related to cost effectiveness in future research [10]. This study will seek to show that peer support models provide a potentially low-cost, flexible compliment to formal health care services [11]. Trained peer leaders can become extensions, or adjuncts to a formal healthcare system, thereby assisting with education delivery and bolstering the efforts of health care professionals [7]. In the current Chinese health care system, diabetes education has not been incorporated, and rural populations with diabetes have not yet acknowledged the responsibility for their own disease or adopted recommended health behaviors. A survey using anonymous standardized patients showed that baseline-educated rural clinicians are not yet prepared to act as the front line fighters in China's primary care system, or equipped to tackle problems in health education [12].Therefore, exploring effective strategies to support diabetes in rural communities is desperately needed.

This article is a study protocol for a cluster randomised controlled trial for a peer support programs in a rural community within China. Our aim is to implement and evaluate a peer support program for people with type 2 diabetes within a specified local rural community. This program is designed to allow the participants to meet and share their self-management challenges, to explore and develop strategies to overcome these challenges, as well as sustain health behavior change using the experience and support derived from the groups. This study will give valuable information about the effectiveness, acceptability and feasibility of a novel way to improve diabetes selfmanagement among developing countries.

\section{Methods}

\section{Objectives}

This study aims to implement and evaluate the biophysical and psychosocial outcomes of a culturally specific peer support program using a cluster randomized controlled trial in patients with type 2 diabetes and to determine whether it is an acceptable, cost effective intervention in the rural community health care centers. The study's objectives are as follows:

1. To establish a culturally appropriate and feasible diabetes peer leader education curriculum by assessing participants' adaptiveness to curriculum components, changes in trainees' knowledge, attitudes, self-care skills and peer support outcomes.

2. To establish a diabetes management network between the hospital, the community, and peer groups, and explore its feasibility and sustainability.

3. To assess the following measures across peer support and control groups: HbA1c, AGE, blood lipids, blood glucose, blood pressure, BMI, medication adherence, knowledge, reported self-efficacy, self-care activities, quality of life, trait affect, life satisfaction, depression, anxiety, and stress.

4. To evaluate differences in cost efficiency between the intervention group and the comparison group.

\section{Study design and setting}

This study is a cluster randomised controlled trial implemented and reported in accordance with the requirements of the CONSORT statement [13] and its extension to cluster randomised trials [14]. The participants were randomly designated to either an intervention arm, which implements the usual care system and the peer support intervention, or a control arm which implements a usual care system, with geographical location being the unit of cluster randomisation. 
All participants are patients with type 2 diabetes who receive primary health care at 12 rural community health centres located in Liu-he District, Nanjing, and Gaogou Town, Huaian, in Jiang Su province. The study team coordinates and works hand-in-hand with the staff members at each community health centres to implement and monitor the study.

Figure 1 is a CONSORT diagram of the study design.

\section{Ethics}

Approval to conduct this study has been granted by the Committee on Human Research (Institutional Review Board) at Zhong Da Hospital, Southeast University (2012 ZDLLKY05.0). Written informed consent was obtained from all trial participants after providing sufficient time to consider partaking in the study, read the details provided on the Patient Information Sheet, in addition to ask and have answered any questions pertaining to the trial.

\section{Eligibility criteria}

\section{Peer leaders}

Inclusion criteria:

1) Patients with type 2 diabetes and HbA1c $\leq 7.5 \%$;

2) Insulin injection experience is preferred,

3) Can commit to attending a 20-hour training, organize activities with other patients every month and attend a leadership meeting at least twice a year,

4) Have basic diabetes self-management knowledge and supportive, non-judgmental communication skills,

5) Willing to lead.
Exclusion criteria:

1) Patients with unstable mood or major psychiatric conditions,

2) Physical disability or severe speech impediment which may influence communication with others,

3) Patients with other serious health conditions (e.g., terminal cancer) or life expectancy less than 12 months,

4) Illiteracy.

\section{Patients}

Inclusion criteria:

1) Patients with type 2 diabetes,

2) Declaration of voluntary participation in the study with signed informed consent form,

3) Reside in local community during the intervention period.

Exclusion criteria:

1) Patients with unstable mood or major psychiatric conditions,

2) Patients with serious diabetes complications (e.g., blindness) that would impede meaningful participation in the program,

3) Patients with other serious health conditions (e.g., terminal cancer) or life expectancy less than 12 months,

4) Patients currently enrolled in other research program.

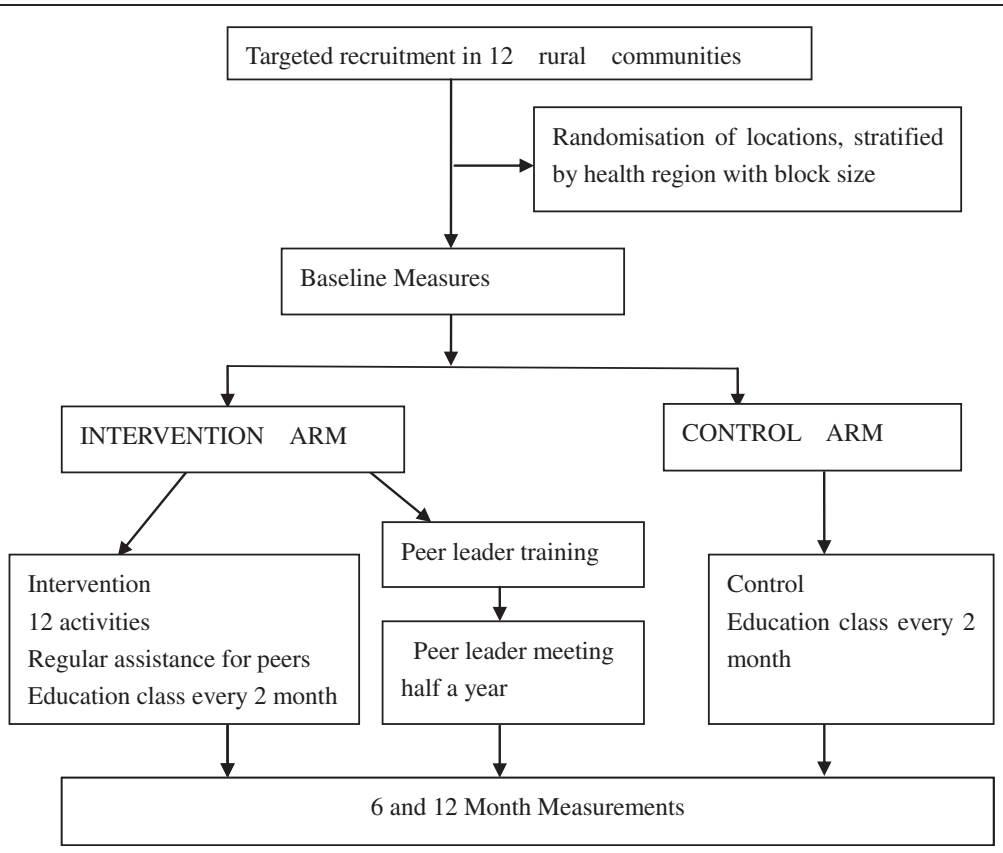

Figure 1 Peer support for patients with type 2 diabetes in Nanjing--Consort diagram for diabetes project study design. 


\section{Enrolment and randomization Participants}

Whether on self-recommendation or recommendation by clinicians and staff members, all peer leader candidates will follow a strict selection procedure from which eligible peer leaders are identified. Additional patients were also selected from primary health care settings and recruited from posted flyers. Primary care clinicians reviewed search results and excluded patients. The study team contacted eligible patients via phone calls to explain the study and arranged enrolment appointments.

\section{Group allocation}

Community health centres will be stratified by size and existing structure of primary diabetes care service. Allocation to intervention or control group is governed by a random number generation process using SAS 9.1 statistical software independently.

\section{Intervention}

\section{Peer leader training}

Peer leaders are required to attend twenty-hour structured training programs in their community which were led by the study team. The training was conducted using a standardised training curriculum developed by the research team. The training team comprised of endocrinology physicians, certified diabetes educators (nurses), registered dieticians, exercise physiologists, psychiatrists, traditional Chinese medicine specialists, and communication trainers. The program aimed at enhancing diabetes knowledge and promoting diabetes self-care behavior. The Peer Leader Training (PLT) curriculum utilizes multiple educational approaches (such as lecture, practise, seminar and etc.) and consists of 5 major components. The content covers basic knowledge of diabetes, behavior change skills, and communication techniques, organisational management methods highlighting individual responsibilities and group management techniques, as well as psychological support.

\section{Control group}

The sessions for the control groups were interactive and informal. Participants were given a handbook that introduces issues in the training session. Patients attend the self-management training and communicate with the professionals every two months to obtain information on diabetes diet, exercise, glucose monitoring, etc. Additionally, group members will attend three follow ups at baseline, 6 months, and 12 months.

\section{Intervention group}

Patients are divided into groups of 8-15 people and assigned to peer leaders for 12 months according to their area of residence. In addition to receiving the same training and follow-ups as the control group, patients from intervention groups were suggested and encouraged to take part in monthly group activities with peer leaders. If possible, casual activities (such as phone calls, WeChat voice messages, physical exercises, group member's family visits, shopping together, etc.) are also recommended. The first meeting is an introductory discussion of group members's backgrounds and their disease history, through which the leaders established understanding and relationship with the peers. In subsequent interactions, the leader and peers will discuss the following issues: their current and target values for HbA1c, blood lipids and blood pressure; self management skills such as using a glucose meter and appropriate strategies for hypoglycaemia; lifestyle changes around healthy diets, physical activities, foot care, and stress control. The subsequent monthly activities include interactions designed by the research team, peer leaders, as well as college student volunteers. Several cultural group activities will be organized such as Chinese hand calisthenics and body calisthenics, how to choose daily nutritional meals, knowledge competition, and singing songs that were integrated with health education topics. Group members also shared similar experiences on lifestyle, family life, personal hobbies and experiences to support each other in achieving goals. Peer leaders documented each encounter and recorded each time the date, number of participants, the nature of encounter, approximate duration of contact, and topics discussed. In addition, peer leader meeting will be held every six months. In the leader meetings, peer leaders will share their experiences and discussed the problems they confronted in their groups. The research team will aid them discover the solutions and provide them with further training when necessary.

\section{Sample size calculation}

Sample size and power calculations are performed for the main outcome of interest-difference in mean HbA1c levels-using effect sizes and standard deviations derived from multiple published trials of patient-education interventions. Using a non-inferiority design based on clinically relevant difference of HbA1c of $0.5 \%$, standard deviation of 1.5 , and groups of $8-15$ participants per randomisation group for $80 \%$ power at two-sided 5\% significance, 150 participants are required to complete the study procedures. Allowing for a $20 \%$ drop-out from randomisation to completion of the trial, it is anticipated that we would require 180 subjects in each arm to be randomised in the study.

\section{Data collection and outcome measures}

Data will be collected at baseline, 6 months and 12 months for both intervention groups and comparison group. Table 1 shows the measurement tools of data collection, 
Table 1 Measurement domains, survey tools used at each data collection time point

\begin{tabular}{|c|c|c|c|c|}
\hline Variable & Measurement tools/questions & Base-line & $6 \mathrm{mth}$ & $12 \mathrm{mth}$ \\
\hline Demographic measures & $\begin{array}{l}\text { Sex, age, ethnicity, religion, education, occupation, health insurance, marital status, } \\
\text { anual income, annual household expenditure }\end{array}$ & $\sqrt{ }$ & & \\
\hline \multirow[t]{2}{*}{ Health status } & $\begin{array}{l}\text { Time of diagnosis, family history, complications and co-morbidities, smoking, alcohol intake, } \\
\text { frequency of physical exercise, length of diagnosis with diabetes, health education access; }\end{array}$ & $\sqrt{ }$ & & \\
\hline & therapy, list of a prescribed medications, events of hypoglycemia & $\sqrt{ }$ & $\sqrt{ }$ & $\sqrt{ }$ \\
\hline \multirow[t]{8}{*}{ Psychosocial status } & Diabetes knowledge & $\sqrt{ }$ & $\sqrt{ }$ & $\sqrt{ }$ \\
\hline & Activities of self-management, (SDSCA) & $\sqrt{ }$ & $\sqrt{ }$ & $\sqrt{ }$ \\
\hline & Self-efficacy (Diabetes Self-Efficacy Scale) & $\sqrt{ }$ & $\sqrt{ }$ & $\sqrt{ }$ \\
\hline & Adherence to medication (Morisky Scale) & $\sqrt{ }$ & $\sqrt{ }$ & $\sqrt{ }$ \\
\hline & Quality of life( EQ5-D, DDS) & $\sqrt{ }$ & $\sqrt{ }$ & $\sqrt{ }$ \\
\hline & Life satisfaction(SWLS) & $\sqrt{ }$ & $\sqrt{ }$ & $\sqrt{ }$ \\
\hline & Trait affect(PANAS) & $\sqrt{ }$ & $\sqrt{ }$ & $\sqrt{ }$ \\
\hline & Depression, anxiety and stress( PHQ-9, DASS) & $\sqrt{ }$ & $\sqrt{ }$ & $\sqrt{ }$ \\
\hline \multirow[t]{7}{*}{ Biomedical measures } & Weight, , height & $\sqrt{ }$ & $\sqrt{ }$ & $\sqrt{ }$ \\
\hline & Waist circumference, hip circumference & $\sqrt{ }$ & $\sqrt{ }$ & $\sqrt{ }$ \\
\hline & Blood pressure, & $\sqrt{ }$ & $\sqrt{ }$ & $\sqrt{ }$ \\
\hline & HbA1c(glycosylated haemoglobin) & $\sqrt{ }$ & $\sqrt{ }$ & $\sqrt{ }$ \\
\hline & AGEs( advanced glycation end products ) & $\sqrt{ }$ & $\sqrt{ }$ & $\sqrt{ }$ \\
\hline & Blood glucose (fasting plasma glucose and 2 hour post load glucose) & $\sqrt{ }$ & $\sqrt{ }$ & $\sqrt{ }$ \\
\hline & Blood Lipids(total cholesterol, low density lipoprotein, high density lipoprotein, triglycerides) & $\sqrt{ }$ & $\sqrt{ }$ & $\sqrt{ }$ \\
\hline Cost & Cost of medicine, travel time and fee, escort fee, hospital stays and fee, cost of services, etc. & $\sqrt{ }$ & $\sqrt{ }$ & $\sqrt{ }$ \\
\hline
\end{tabular}

the method of data collection and the time points of data collection.

Data collection consists of two main parts, questionnaire and biophysical data. The questionnaire data includes four main parts and will be collected by trained investigators. The first part covers social demographics (e.g., sex, age, ethnicity, religion, education level, occupation, health insurance, marital status, annual income, etc.); the second portion concerns measures of health status (eg, time of diagnosis, smoking, alcohol intake, frequency of physical exercise, therapy, length of diagnosis with diabetes, family history, complications and co-morbidities, educational history and type, glucose monitor). The third section is psychosocial status including knowledge of diabetes; activities of self-management [15], self-efficacy [16], adherence to medication $[17,18]$, quality of life [19-21], diabetes distress screening [22,23], traits [24], life satisfaction [25], depression, anxiety and stress [26-28]. The final section asks about economic status, which covers training, equipment availability, human resources, drugs, travel fees, escort fees, communication expenses, consumables, etc.

The biophysical data are collected by specifically trained staff according to standard operation procedure. Height is measured using a portable height rod to the nearest $0.1 \mathrm{~cm}$ with the participants standing without headgear or shoes. Weight is measured to the nearest $0.1 \mathrm{~kg}$ using a calibrated, portable, digital weighing scale. Body mass in dex (BMI) is calculated as weight in kilograms divided by height in meters squared. Waist circumference is measured midway between the lowest rib and the top of the hip bone using the tape measure. Hip circumference is taken at the maximum circumference over the buttocks [29]. Duplicate waist and hip circumference measurement is taken for approximately $10 \%$ of the participants for quality assurance. Blood pressure meter and weight scales are calibrated at least weekly. Laboratories providing blood analysis are accredited, and all methods for determining HbA1c\% are NGSP approved.

\section{Outcome measures}

Outcomes variables are measured at baseline, 6 months, and 12 months, to both participants and peer leaders. The primary expected outcome is the change in $\mathrm{HbA1c}$ at baseline compared to the sixth month and baseline to the twelfth month. HbA1c, a measurement of glycosylated haemoglobin, is regarded as the gold standard measure of glycemic control reflecting overall blood glucose values over the previous $8-12$ weeks [30,31]. There is a strong relationship between HbA1c and the risk of developing long-term diabetic complications and it is accepted as a reasonable surrogate for long-term outcomes in individuals with diabetes. Secondary outcomes are advanced glycation end products (AGEs) [32,33], blood pressure, BMI, blood lipids, blood glucose (fasting plasma glucose 
and 2 hour post load glucose), patient-reported rates of severe hypoglycaemia, diabetes self-care activities, medication adherence, knowledge, quality of life, diabetes selfefficacy, traits, life satisfaction, depression, anxiety, and stress. In addition, cost is also assessed.

\section{Data analysis}

Social demographic data and health status of the study subjects are presented as frequencies (percentage) for categorical variables, and means \pm standard deviations for continuous variables. Comparison of patients characteristics between groups will be checked by means of Ttests or $\chi^{2}$ tests, or non-parametric equivalents. Repeated measures analysis of variance and multilevel analysis for longitudinal data will be used to examine longitudinal differences between groups on primary and secondary outcome measures. Evaluation of intervention effectiveness will be by intention to treat using the above statistical tests. Evidence of clustering by clinic site and primary care provider will be examined and adjusted for analyses as needed. If significant differences in baseline characteristics are found, analyses will be repeated adjusting for these differences using ANOVA and logistic regression for multivariate analyses. Analysis of covariance (i.e. linear regression models for 12-month measurements with baseline scores and intervention arm as covariates) will estimate the changes from baseline to 12 months that can be attributed to the peer support intervention. Sensitivity analyses will be performed to estimate the effects of missing data using different assumptions (e.g. imputed values). The EuroQoL 5 dimensional health state measure (EQ5D) score at base-line and follow-up can be converted into utility weights using the EQ5D algorithm [34], which then allow for the calculation of quality adjusted life years (QALYs). Economic analyses will be employed to estimate direct and indirect cost analysis, the cost per QALY gained and incremental cost-effectiveness ratios. Sensitivity analysis will be undertaken to test the stability of the analysis in terms of the cost inputs and health outcomes. All analyses will be carried out with SPSS 10.0 software.

\section{Qualitative evaluation}

A descriptive parallel qualitative analysis is being carried out to record the attitude of patients, professionals, educators, rural clinicians and peer leaders to peer support and their experience of delivery in intervention practices. The peer leaders will voluntarily participate in a focus group, semi-structured interviews, or both, to assess how they experience the training and leading process. The focus group is designed to reveal the peer leaders' general attitude and facilitate the development of the interview guide. Peer leaders are encouraged to discuss their feelings and efficacy, the training experience, motivations, as well as any positive and negative impacts on their own diabetes management. Semi-structured qualitative interviews and focus group with diabetic patients will also be carried out and analysed through the investigation and daily observations. The focus group and interviews are audio recorded and transcribed.

\section{Discussion}

The World Health Organization's action plan for chronic disease management helps encouraged governments to take action to aid people to better managing their own chronic conditions by providing education, incentives, self-management tools and care [35]. Implementation of a diabetes management plan should include clinical care, diabetes self-management education and ongoing support [36]. Self-management training and support is an effective component of care for people with diabetes. Due to a shortage of time and resources, this critical support is not consistently delivered in most health care settings. The study uses trained patients to provide peer support in urban community. We anticipate that patients in the intervention arm with a peer leader will show significant improvement in the biophysical and psychosocial outcomes, and so will the peer leaders. In addition, we hypothesize that the recruitment and training of volunteers with type 2 diabetes as peer leaders to support participants with the same conditions will be a cost-effective strategy. We expect that the qualitative and health economic research will reveal an in-depth perspective on the impact of this approach both at the level of the patient as an individual and at the societal level. Successful implementation of this trial will provide evidence of the key functions of peer support, identified by the global Peers for Progress program. Moreover, the results of this study will influence future policies on diabetes management, as well as other chronic diseases in China.

\section{Competing interest}

The study was supported by small grant of peers for progress, Jiangsu Research Funds of PhD student Program and the Fundamental Research Funds for the Central Universities.

\section{Authors' contributions}

Principal Investigators SZ conceived the proposal, obtained funding and contributed to the study design, manuscript preparation and approved the final draft. $B X, X Y, M J, Y L, V C$ participated in the study design and prepared the first manuscript. CJ, YY participated in funding application, study design, and discussion of early drafts. HJ contributed economic analysis design and data collection methods. All authors reviewed and agreed the final version of this manuscript. All authors read and approved the final manuscript.

\section{Acknowledgements}

The authors acknowledge the contributions of Prof. BW in the development of the study protocol and Dr. HJ, JH. QL, LL, TY, XC and other colleagues in the piloting, development and delivery of the peer leader training program. We acknowledge the peer leaders who would like to participate in this program for their enthusiasm and dedication to the study. We thank the student volunteers in Southeast University for project assistance. We are truly indebted to Peers for Progress, a global initiative funded by the American Academy of Family

Physicians Foundation for their great help and technical support. 


\section{Author details}

'Department of Endocrinology, Zhongda Hospital, Institute of Diabetes, Medical School, Southeast University, Nanjing 210009, China. ²Department of Epidemiology and Health Statistics, Southeast University, Nanjing 210009, China.

Received: 31 May 2014 Accepted: 16 July 2014

Published: 23 July 2014

\section{References}

1. Guariguata L, Whiting DR, Hambleton I, Beagley J, Linnenkamp U, Shaw JE: Global estimates of diabetes prevalence for 2013 and projections for 2035. Diabetes Res Clin Pract 2014, 103(2):137-149.

2. Zhang P, Zhang X, Brown J, Vistisen D, Sicree R, Shaw J, Nichols G: Global healthcare expenditure on diabetes for 2010 and 2030. Diabetes Res Clin Pract 2010, 87(3):293-301.

3. Zabetian A, Sanchez IM, Narayan KM, Hwang CK, Ali MK: Global rural diabetes prevalence: A systematic review and meta-analysis covering 1990-2012. Diabetes Res Clin Pract 2014, 104(2):206-213.

4. Routasalo PE, Savikko N, Tilvis RS, Strandberg TE, Pitkala KH: Social contacts and their relationship to loneliness among aged people - a population-based study. Gerontology 2006, 52(3):181-187.

5. Ghorob A, Vivas MM, De Vore D, Ngo V, Bodenheimer T, Chen E, Thom DH: The effectiveness of peer health coaching in improving glycemic control among low-income patients with diabetes: protocol for a randomized controlled trial. BMC Public Health 2011, 11:208.

6. Xu Y, Wang L, He J, Bi Y, Li M, Wang T, Wang L, Jiang Y, Dai M, Lu J, Xu M, Li Y, Hu N, Li J, Mi S, Chen CS, Li G, Mu Y, Zhao J, Kong L, Chen J, Lai S, Wang W, Zhao W, Ning G, 2010 China Noncommunicable Disease Surveillance Group: Prevalence and control of diabetes in Chinese adults. JAMA 2013, 310(9):948-959.

7. Shen H, Edwards H, Courtney M, McDowell J, Wu M: Peer-led diabetes self-management programme for community-dwelling older people in China: study protocol for a quasi-experimental design. J Adv Nurs 2012, 68(12):2766-2777.

8. Dennis C-L: Peer support within a health care context: a concept analysis Int J Nurs Stud 2003, 40(3):321-332.

9. Boothroyd RI, Fisher EB: Peers for progress: promoting peer support for health around the world. Fam Pract 2010, 27(Suppl 1):i62-i68.

10. Dale JR, Williams SM, Bowyer V: What is the effect of peer support on diabetes outcomes in adults? A systematic review. Diabet Med 2012, 29(11):1361-1377.

11. Heisler M: Different models to mobilize peer support to improve diabetes self-management and clinical outcomes: evidence, logistics, evaluation considerations and needs for future research. Fam Pract 2010, 27(Suppl 1):i23-i32.

12. Sylvia S, Shi Y, Xue H, Tian X, Wang H, Liu Q, Medina A, Rozelle S: Survey using incognito standardized patients shows poor quality care in China's rural clinics. Health Policy Plan 2014, [Epub ahead of print].

13. Moher D, Hopewell S, Schulz KF, Montori V, Gotzsche PC, Devereaux PJ, Elbourne D, Egger M, Altman DG: CONSORT 2010 Explanation and Elaboration: updated guidelines for reporting parallel group randomised trials. BMJ 2010, 340(mar23 1):C869-c879.

14. Campbell MK, Piaggio G, Elbourne DR, Altman DG: Consort 2010 statement: extension to cluster randomised trials. BMJ 2012 345(sep04 1):e5661-e5671.

15. Toobert DJ, Hampson SE, Glasgow RE: The summary of diabetes self-care activities measure: results from 7 studies and a revised scale. Diabetes Care 2000, 23(7):943-950.

16. Lorig K, Ritter PL, Villa FJ, Armas J: Community-based peer-led diabetes self-management: a randomized trial. Diabetes Educ 2009, 35(4):641-651.

17. Morisky DE, Ang A, Krousel-Wood M, Ward HJ: Predictive validity of a medication adherence measure in an outpatient setting. $J$ Clin Hypertens (Greenwich) 2008, 10(5):348-354.

18. Morisky DE, Green LW, Levine DM: Concurrent and predictive validity of a self-reported measure of medication adherence. Med Care 1986, 24(1):67-74

19. Koltowska-Haggstrom $M$, Jonsson $B$, Isacson D, Bingefors $K$ : Using EQ-5D to derive general population-based utilities for the quality of life assessment of growth hormone deficiency in adults (QoL-AGHDA). Value Health 2007, 10(1):73-81.
20. Wang HM, Patrick DL, Edwards TC, Skalicky AM, Zeng HY, Gu WW: Validation of the EQ-5D in a general population sample in urban China Qual Life Res 2012, 21(1):155-160

21. EuroQol Group: EuroQol-a new facility for the measurement of health-related quality of life. Health Policy 1990, 16(3):199-208.

22. Fisher L, Glasgow RE, Mullan JT, Skaff MM, Polonsky WH: Development of a brief diabetes distress screening instrument. Ann Fam Med 2008, 6(3):246-252.

23. Polonsky WH, Fisher L, Earles J, Dudl RJ, Lees J, Mullan J, Jackson RA: Assessing psychosocial distress in diabetes: development of the diabetes distress scale. Diabetes Care 2005, 28(3):626-631.

24. Thompson ER: Development and validation of an internationally reliable short-form of the Positive and Negative Affect Schedule (PANAS). J Cross Cult Gerontol 2007, 38(2):227-242

25. Diener E, Emmons RA, Larsen RJ, Griffin S: The Satisfaction With Life Scale. J Pers Assess 1985, 49(1):71-75.

26. Kroenke K, Spitzer RL, Williams JB: The PHQ-9: validity of a brief depression severity measure. J Gen Intern Med 2001, 16(9):606-613.

27. Lovibond PF, Lovibond SH: The structure of negative emotional states: comparison of the Depression Anxiety Stress Scales (DASS) with the Beck Depression and Anxiety Inventories. Behav Res Ther 1995, 33(3):335-343.

28. Chan RC, Xu T, Huang J, Wang Y, Zhao Q, Shum DH, O'Gorman J, Potangaroa R: Extending the utility of the Depression Anxiety Stress scale by examining its psychometric properties in Chinese settings. Psychiatry Res 2012, 200(2-3):879-883.

29. The WHO STEPwise approach to surveillance (STEPS). [http://www.who. int/chp/steps/en/]

30. Hadden DR, Savage GA, Lappin TR, Elder GE: What is the gold standard for glycosylated haemoglobin? Lancet 1993, 342(8884):1424-1425.

31. Peters AL, Davidson MB, Schriger DL, Hasselblad V: A clinical approach for the diagnosis of diabetes mellitus: an analysis using glycosylated hemoglobin levels. Meta-analysis Research Group on the Diagnosis of Diabetes Using Glycated Hemoglobin Levels. JAMA 1996, 276(15):1246-1252.

32. Makita Z, Radoff S, Rayfield EJ, Yang Z, Skolnik E, Delaney V, Friedman EA Cerami A, Vlassara H: Advanced glycosylation end products in patients with diabetic nephropathy. N Engl J Med 1991, 325(12):836-842.

33. Brownlee $\mathrm{M}$, Cerami A, Vlassara $\mathrm{H}$ : Advanced glycosylation end products in tissue and the biochemical basis of diabetic complications. N Engl J Med 1988, 318(20):1315-1321.

34. Lamers LM, McDonnell J, Stalmeier PF, Krabbe PF, Busschbach JJ: The Dutch tariff: results and arguments for an effective design for national EQ-5D valuation studies. Health Econ 2006, 15(10):1121-1132

35. World Health Organization: 2008-2013 action plan for the global strategy for the prevention and control of noncommunicable diseases. Geneva: World Health Organization; 2009.

36. Amer Diabet A: Standards of medical care in diabetes-2011 American Diabetes Association. Diabetes Care 2011, 34:S11-S61.

\section{doi:10.1186/1471-2458-14-747}

Cite this article as: Xie et al:: Peer support for patients with type 2 diabetes in rural communities of China: protocol for a cluster randomized controlled trial. BMC Public Health 2014 14:747.

\section{Submit your next manuscript to BioMed Central and take full advantage of:}

- Convenient online submission

- Thorough peer review

- No space constraints or color figure charges

- Immediate publication on acceptance

- Inclusion in PubMed, CAS, Scopus and Google Scholar

- Research which is freely available for redistribution 